\title{
Endovascular exclusion of a false lumen associated with residual dissection after proximal aortic repair using a custom-made false lumen occluder
}

Yuhei Saitoh, MD, PhD, ${ }^{\mathrm{a}}$ Yoshihiko Yokoi, MD, ${ }^{\mathrm{b}}$ Takeshi Soeda, MD, ${ }^{\mathrm{a}}$ and Kouichi Yuri, MD, ${ }^{\mathrm{c}}$ Matsue, Tokyo, and Saitama, Japan

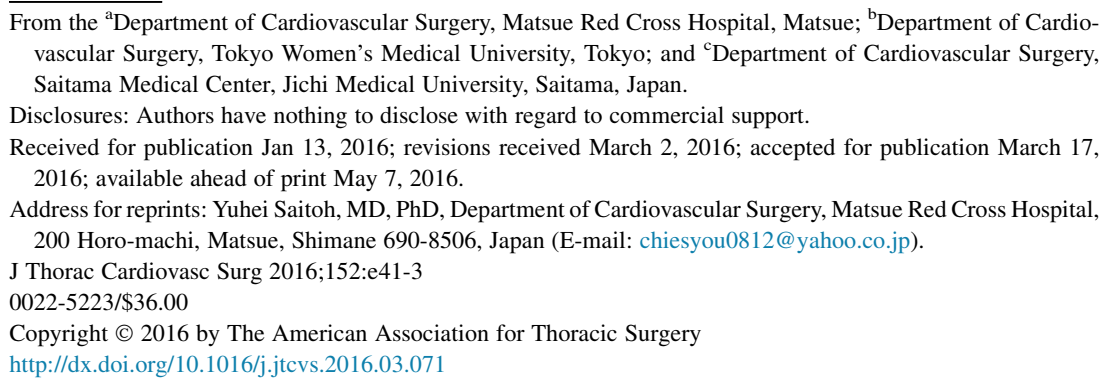

The choice of surgical treatment for chronic dissection of the descending aorta is a challenging problem considering its high morbidity. The effectiveness and safety of thoracic endovascular aortic repair (TEVAR) for aortic dissection have been confirmed in previous studies. ${ }^{1,2}$ Achieving

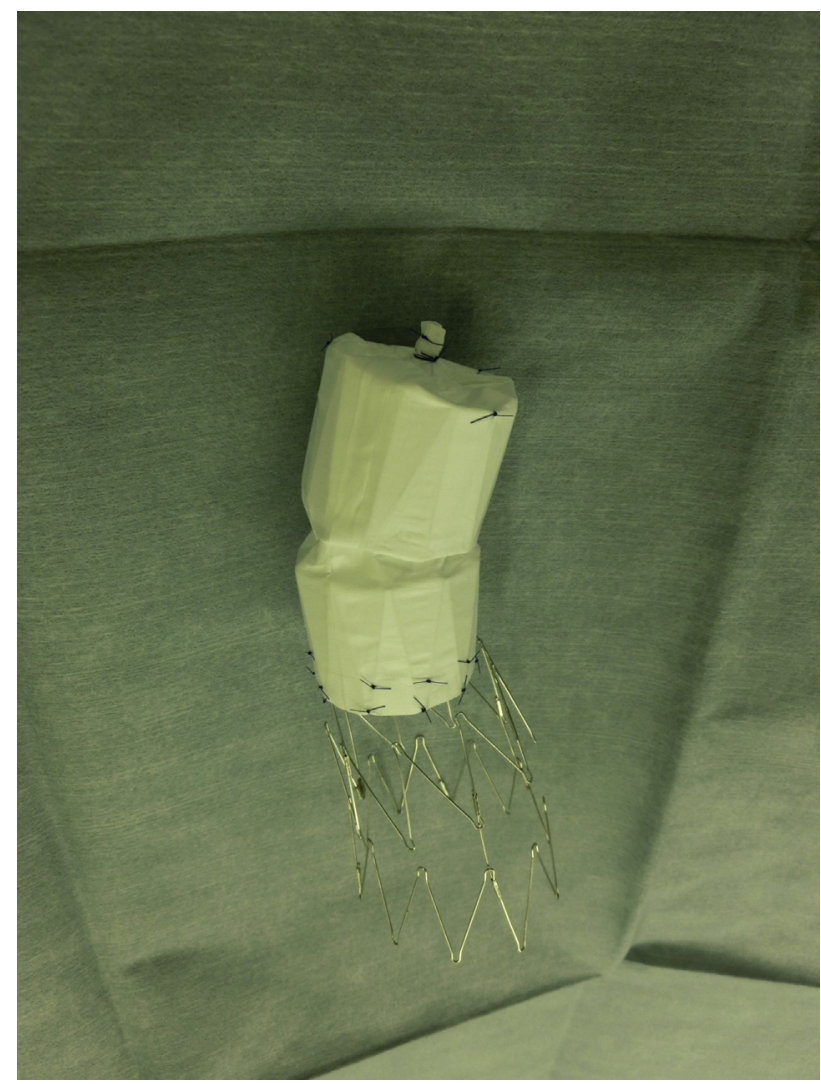

FIGURE 1. The occluder consisted of an expanded polytetrafluoroethylene graft with a closed top of the graft.

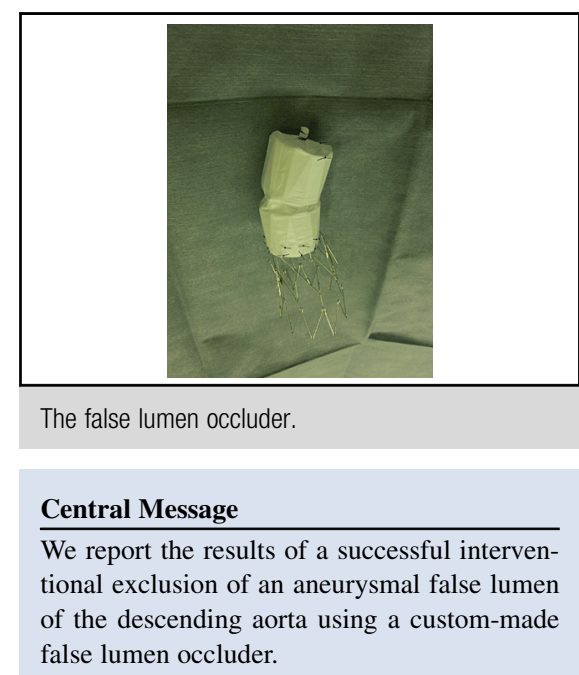

See Editorial Commentary page e45.

complete thrombosis of the false lumen is difficult, however, because most patients with chronic dissection have 1 or more reentry tears, which provide persistent flow and blood pressure to the false lumen.

Here we report the results of a successful interventional exclusion of an aneurysmal false lumen of the descending aorta using a custom-made false lumen occluder (Figure 1).

\section{CLINICAL SUMMARY}

A 61-year-old woman underwent ascending aorta replacement because of acute type A aortic dissection that occurred at age 54 years. Residual dissection from the aortic arch to the terminal aorta and dilation of the thoracic descending aorta were detected by computed tomography (CT) scan. Debranching TEVAR was performed to prevent aortic rupture. After bypass surgery using a prosthesis between the ascending aorta and the supra-aortic vessels, a precurved tapered stent graft (Najuta, 30/26 × $200 \mathrm{~mm}$; Kawasumi Laboratories, Tokyo, Japan) was implanted from the prosthesis to the thoracic descending aorta. Although intraoperative angiography showed complete exclusion of the primary entry site, the false lumen of the descending aorta was still enhanced on a postoperative CT scan.

At 4 months after TEVAR, a CT scan showed increased false lumen enhancement of the thoracic descending aorta 


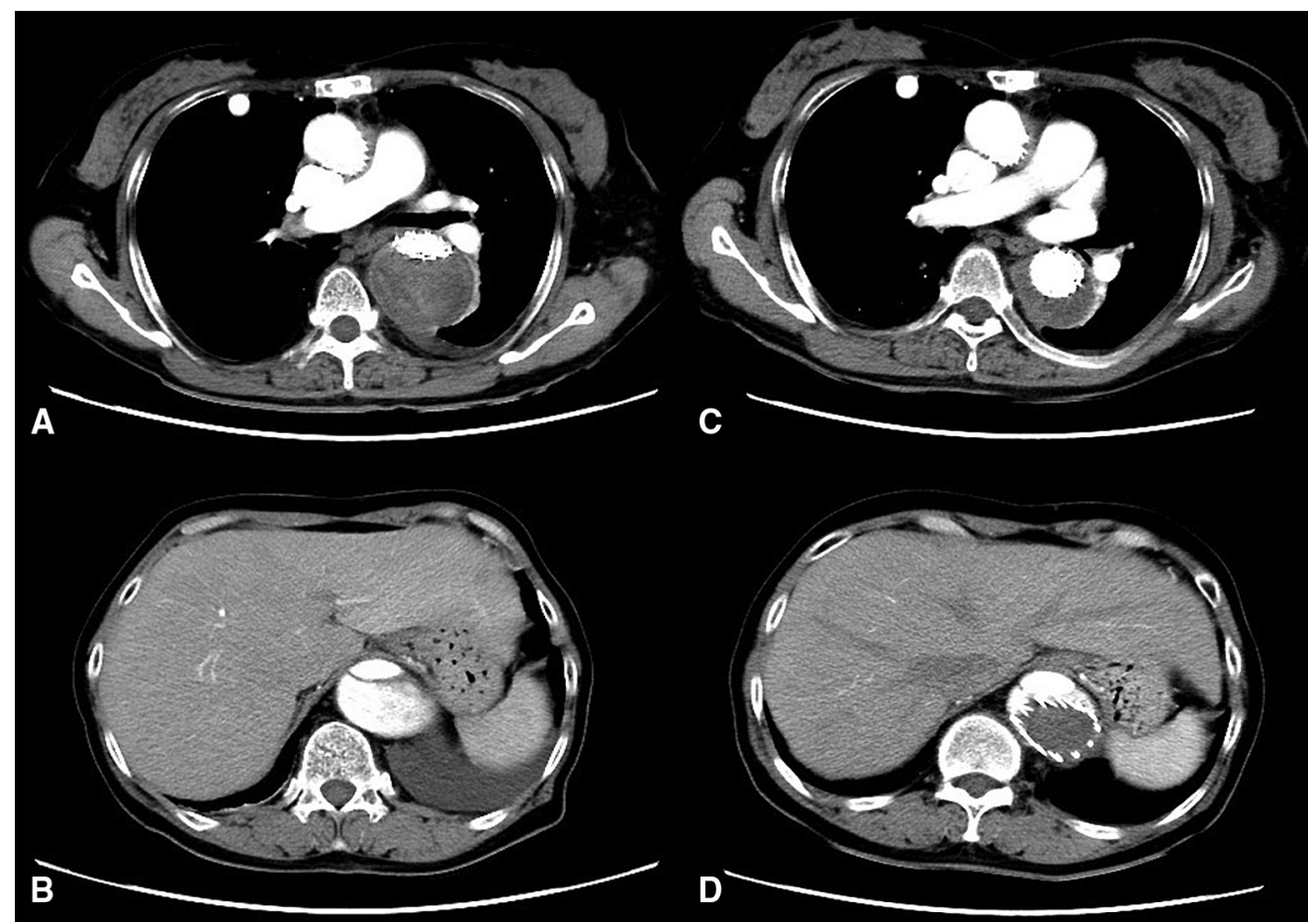

FIGURE 2. A and B, CT scan before implantation of the occluder showing narrowing of the true lumen and a widely enlarged false lumen. C and D, Followup CT at 9 months after implantation of the false lumen occluder showing remodeling of the thoracic descending aorta and significant shrinking of the false lumen (C), but no migration of the occluder (D).

(Figures 2, $A$ and $B$, and 3, $A$ ). A second intervention was performed to decrease the retrograde flow from reentry into the abdominal aorta. First, a 20 Fr Flexor KellerTimmermans introducer (Cook Medical, Bloomington,
Ind) was inserted into the false lumen of the thoracic descending aorta through a reentry at the left common iliac artery. Then the custom-made false lumen occluder was pushed up through the sheath and deployed to the false
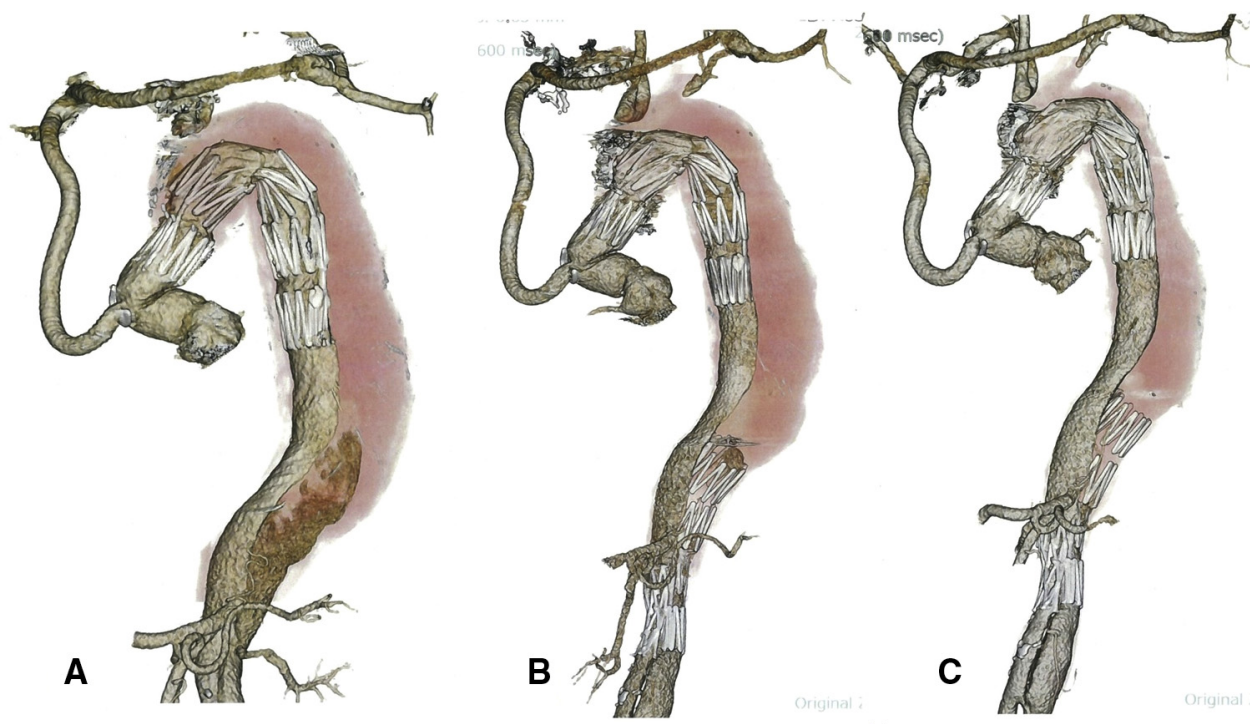

FIGURE 3. A, CT scan after debranching TEVAR showing narrowing of the true lumen and major enlargement of the false lumen. B, View after implantation of the false lumen occluder at 10 days after the operation. Retrograde flow to the false lumen was blocked by the occluder and clearly disappeared. C, Follow-up CT at 9 months after implantation of the false lumen occluder showing remodeling of the thoracic descending aorta and significant shrinking of the false lumen. Migration of the occluder was not observed. 
lumen at the proximal side of the celiac artery branch. This implant consisted of an expandable polytetrafluoroethylene graft and stainless steel stent skeleton with a closed top of the graft and the lower half of the bared stent. The diameter of the device was determined by 3-dimensional CT, which directly measured the outline of the deformed false lumen.

Next, after changing to insert the stiff wire into the true lumen, a Powerlink limb extension $(20 \mathrm{~mm} \times 55 \mathrm{~mm})$ was deployed on the true lumen of the infrarenal abdominal aorta to close the reentries and secure perfusion to the true lumen. Finally, coil embolization of the minor entry to the aortic arch was performed via the right brachial artery.

A postoperative CT scan revealed no enhancement of the false lumen on the thoracic descending aorta (Figure 3, B), and the patient was discharged once recovery was complete.

A follow-up CT scan performed at 9 months after the additional operation showed remodeling of the thoracic descending aorta and significant shrinking of the false lumen (Figures 2, $C$ and $D$, and 3, $C$ ).

\section{DISCUSSION}

The purpose of endografting for aortic dissection to close primary or major entry tears is to prevent enlargement of the false lumen and avoid aortic rupture. Nonetheless, the false lumen sometimes enlarges continuously, because almost all types of chronic aortic dissection (eg, Stanford type B dissection, residual dissection after repair of Stanford type A dissection) have multiple reentries that perfuse the false lumen. In such a situation, endografting may prove difficult, because reentry tears tend to be located near the branch vessels of the abdominal aorta, and some important organs are perfused from the false lumen. There have been several few reports of the effective treatment of reentry tears for type B aortic dissection with a vascular occluder made for peripheral arteries. ${ }^{3-5}$

In this case, enlargement of the thoracic false lumen was prevented by using the endovascular occlusion technique without organ ischemia. The thoracic false lumen was isolated from the abdominal false lumen using a false lumen occluder. Adequate blood pressure reduction in the thoracic false lumen was obtained with this less invasive method.

Although it is not necessary to close the false lumen when all identified reentry tears have been closed, identifying multiple reentry tears in the residual dissected aorta is difficult in practice. Moreover, closure of all of the reentry tears might lead to visceral and spinal cord ischemia. The false lumen occluder leaves several reentries in the abdominal aorta to maintain blood flow to major organs from both the true and false lumens. This procedure is a safer approach to treating complex chronic dissection. Long-term follow-up is needed to evaluate the efficacy and durability of this approach.

\section{References}

1. Nienaber CA, Fattori R, Lund G, Dieckmann C, Wolf W, von Kodolitsch Y, et al. Nonsurgical reconstruction of thoracic aortic dissection by stent-graft placement. N Engl J Med. 1999;340:1539-45.

2. Dake MD, Kato N, Mitchell RS, Semba CP, Razavi MK, Shimono T, et al. Endovascular nonsurgical stent-graft placement for the treatment of acute aortic dissection. N Engl J Med. 1999;340:1546-52.

3. Tang X, Fu W, Xu X, Yang J, Shi Y, Yan Z, et al. Use of a vascular occluder to treat a re-entry tear in a patient with Stanford type B aortic dissection: acute and 1-year results. J Endovasc Ther. 2008;15:566-9.

4. Loubert MC, van der Hulst VP, Vries CD, Bloemendaal K, Vahl AC. How to exclude the dilated false lumen in patients after a type B aortic dissection? The cork in the bottleneck. J Endovasc Ther. 2003;10:244-8.

5. Shi H, Lu M, Jiang M. Use of a stent-graft and vascular occlude to treat primary and re-entry tears in a patient with a Stanford type B aortic dissection. J Rev Bras Cir Cardiovasc. 2013;28:550-4. 